\title{
Response of eyes with age-related macular degeneration to anti-VEGF drugs and implications for therapy planning
}

This article was published in the following Dove Press journal:

Clinical Ophthalmology

28 April 2017

Number of times this article has been viewed

\author{
Noriko Miyamoto ${ }^{1,2}$ \\ Michiko Mandai ${ }^{1,3}$ \\ Hiroshi Kojima ${ }^{1,2}$ \\ Takanori Kamedal,2 \\ Masataka Shimozono ${ }^{1,2}$ \\ Akihiro Nishida ${ }^{1,2}$ \\ Yasuo Kurimoto ${ }^{1,2}$ \\ 'Department of Ophthalmology, Kobe \\ City Medical Center General Hospital, \\ ${ }^{2}$ Department of Ophthalmology, \\ Institute of Biomedical Research and \\ Innovation, ${ }^{3}$ Laboratory for Retinal \\ Regeneration, RIKEN Center for \\ Developmental Biology, Kobe, Japan
}

Purpose: To evaluate the response to and dependence on aflibercept or ranibizumab in patients with age-related macular degeneration (AMD).

Methods: We retrospectively reviewed AMD patients who received induction therapy with aflibercept or ranibizumab for the following parameters: whether complete resolution of the retinal fluid ("good response") was achieved and whether recurrence was observed within 3 months ("dependent") after the induction treatment. With aflibercept treatment, treatmentnaïve eyes with a good response/non-dependence were recommended a pro re nata regimen, and other eyes were recommended a proactive bimonthly regimen, followed by monitoring of visual acuity (VA) for 12 months. The measured values of the groups were compared using one-way analysis of variance with Tukey's test to evaluate the difference between baseline and postinjection VA.

Results: Among the treatment-naïve eyes, $76 \%$ had a good response to aflibercept and $37 \%$ of these were aflibercept-dependent, while $58 \%$ had a good response to ranibizumab but $51 \%$ of these were ranibizumab-dependent. Among the eyes that converted from ranibizumab treatment, $92 \%$ of the good responders to ranibizumab with dependence and $76 \%$ of the poor responders on ranibizumab had a good response to aflibercept. With aflibercept treatment, the mean VA of treatment-naïve patients was significantly better than the baseline VA over 12 months $(P<0.001)$, and the VA of the converted group improved significantly with proactive treatment and the improvement was continuously maintained from 6 to 12 months.

Conclusion: The evaluation of response to and dependence on anti-vascular endothelial growth factor therapies in AMD was useful and practical in managing therapeutic protocols to obtain a good VA.

Keywords: aflibercept, ranibizumab, recurrence, dependence, visual acuity, proactive treatment

\section{Introduction}

Neovascular exudative age-related macular degeneration (AMD) can cause significant, acquired vision loss in elderly patients. Intravitreal bevacizumab and ranibizumab injections have been routinely used as anti-vascular endothelial growth factor (VEGF) agents for AMD, and many randomized studies have reported that these agents have a significant impact on preserving vision and preventing blindness. ${ }^{1-3}$

Aflibercept has been approved recently as an anti-VEGF drug. It binds to members of the VEGF family, including all VEGF-A and VEGF-B isoforms, as well as to placental growth factor (PlGF), ${ }^{4}$ and maintains significant intravitreal VEGF-binding activity for longer periods than ranibizumab in AMD patients. ${ }^{5,6}$ It has also been reported that bevacizumab and ranibizumab had equivalent effects on visual acuity
Correspondence: Noriko Miyamoto Department of Ophthalmology, Kobe City Medical Center General Hospital, 2-I-I Minatojima-Minamimachi, Chuo-ku, Kobe 6500047, Hyogo, Japan

$\mathrm{Tel}+8 \mathrm{I} 78302432$ I

Fax +8I 783022487

Emailmiyafrac@kcho.jp (c)
hereby accept the Terms. Non-commercial uses of the work are permitted without any further permission from Dove Medical Press Limited, provided the work is properly attributed. For permission for commercial use of this work, please see paragraphs 4.2 and 5 of our Terms (https://www.dovepress.com/terms.php). 
(VA) at 1 year, ${ }^{2}$ and that aflibercept therapy was beneficial for AMD patients who exhibited recurrent or resistant exudative changes following multiple injections with either bevacizumab or ranibizumab..$^{7-12}$

It is now widely accepted that a 3-month induction therapy with anti-VEGF drugs, ranibizumab, or aflibercept improves VA, but continuous intensive treatment such as monthly ranibizumab or bimonthly aflibercept is essential to maintain this improved VA (MARINA, ${ }^{1}$ ANCHOR, ${ }^{3}$ and VIEW ${ }^{13}$ ). Intensive treatment with anti-VEGF drugs could be a costly and physical burden for aged patients. The PrONTO study ${ }^{14}$ reported a successful reduction in the number of injections by introducing a strict pro re nata (PRN) regimen with monthly monitoring that successfully maintained the improved VA and the decreased central retinal thickness (CRT) at the end of a 2-year induction therapy with the average of 5.6 injections (for the first year). The PrONTO study also demonstrated that the mean injection-free interval was 4.5 months before another injection was necessary, meaning some of these intensive treatments provided overtreatment.

The objective of this study was to 1) retrospectively review the overall rates of good response and dependence of treatment-naïve AMD patients who received induction therapies with intravitreal injections of aflibercept or ranibizumab to estimate the population that may require intensive treatment, and 2) determine if the information on response/dependence may help therapeutic planning without impairing visual outcomes. Additionally, patients who were converted to aflibercept due to either a poor response or dependence with a good response from ranibizumab were also studied for response to aflibercept induction therapy. We further investigated the possible factors that affected these parameters. Lastly, we reviewed the VA outcomes of the current regular protocol used in our hospital, which used proactive aflibercept injection for the cases with insufficient response and early recurrence. Although the study was retrospective, we postulated that response and dependence could be useful parameters when establishing plans for antiVEGF therapies.

\section{Patients and methods}

\section{Patients}

We retrospectively reviewed the medical records of all AMD patients who received induction therapy with aflibercept (159 eyes of 146 patients) between February 2013 and July 2014 and with ranibizumab (77 eyes of 72 treatment-naïve patients) between June 2009 and December 2011 at Kobe City Medical Center General Hospital.
Of the aflibercept-treated patients, 71 eyes of 66 patients were treatment-naïve, and 88 eyes of 80 patients were converted from ranibizumab to aflibercept. Patients who did not complete 12 months of follow-up were not included in this study. Patients treated with aflibercept were reviewed over 1 year for VA and CRT. We also studied the possible pretreatment factors that would affect the response to or dependence on aflibercept. All procedures conformed to the Declaration of Helsinki for research involving human subjects, and approval was obtained from the Institutional Review Board of the Kobe City Medical Center General Hospital. The review board waived the need for written informed consent because the study design consisted of a retrospective chart review.

\section{Treatment and study design}

All the eyes were treated using a 3-month induction therapy with aflibercept $(2 \mathrm{mg} / 0.05 \mathrm{~mL})$ or ranibizumab $(0.05 \mathrm{mg} / 0.05 \mathrm{~mL})$. A good response was defined as complete resolution of intraretinal fluid (IRF) and subretinal fluid (SRF) measured using spectral domain optical coherence tomography (SD-OCT) (Spectralis ${ }^{\circledR}$ HRA + OCT; Heidelberg Engineering, Carlsbad, CA, USA) at 1 month after the end of anti-VEGF induction therapy. The eyes with good responses were monitored for relapses with any fluid appearance, and were defined as "treatment dependent" if the retinal fluid recurred within 3 months from the end of the induction therapy.

Treatment-naïve AMD eyes were treated with a 3-month induction therapy using aflibercept, and all the eyes with a good response were assigned a PRN regimen. Treatmentnaïve eyes that responded insufficiently, or that had a good response but were dependent on aflibercept, were assigned a proactive bimonthly regimen. We reviewed the group of patients converted to aflibercept due to an insufficient response to or being dependent on ranibizumab. We also investigated the exudative change-free survival percentages of treatment-naïve AMD eyes after aflibercept treatment and compared them with those of good responders to ranibizumab according to Kaplan-Meier survival analyses. In the converted group, all the patients were recommended bimonthly proactive aflibercept during the maintenance phase after induction therapy. In all cases, the patients were informed of the PRN and bimonthly treatment regimen, and were free to select either regimen. The patients were followed by monthly monitoring as was the case in the PrONTO study, ${ }^{14}$ and the retreatment criteria were applied as any fluid observed using OCT for patients treated with aflibercept on the PRN regimen. 
As suggested, we applied the PrONTO study procedures for patients treated with ranibizumab.

\section{Outcomes}

SD-OCT images were obtained with a Spectralis ${ }^{\circledR}$ OCT machine (Spectralis ${ }^{\circledR}$ Family Acquisition Module, version 4.0.2.0; Heidelberg Engineering) and Heidelberg Eye Explorer (version 1.6.1.0; Heidelberg Engineering) before induction therapy and every month thereafter. Horizontal and vertical scans of the macula were recorded for each eye. The measurements were performed under pupillary dilation. CRT was defined as the distance between the vitreoretinal border and the edge of the retinal pigment epithelial cells. We determined the presence or absence of pigment epithelial detachment (PED), IRF, and SRF. These procedures were performed within a foveal area of $1.8-\mathrm{mm}$ diameters, which is considered to be the macula. ${ }^{15} \mathrm{VA}$ was measured with a Landolt $\mathrm{C}$ chart and then converted to a logarithm of the minimum angle of resolution (logMAR) equivalent. Clinical characteristics, including age, sex, greatest linear dimension (GLD), and disease subtypes such as typical AMD, polypoidal choroidal vasculopathy (PCV), and retinal angiomatous proliferation, were also reviewed and analyzed. We also studied the possible pretreatment factors (age, sex, subtype, VA, CRT, GLD, PED, IRF, SRF, and treatment history) that would affect the response to or dependence on aflibercept.

\section{Statistical analyses}

The statistical program SPSS (version 11.0.1; SPSS Japan, Tokyo, Japan) was used to analyze the sample data. Descriptive analyses are reported as the mean \pm standard deviation unless otherwise specified. The chi-square test was used to compare the baseline clinical characteristics between aflibercept and ranibizumab, and between the treatment-naïve for aflibercept and converted to aflibercept groups. The measured values of the groups were compared using one-way analysis of variance with Tukey's test to evaluate the difference between baseline and postinjection (from 3 to 12 months) VA and CRT results. Kaplan-Meier analyses were used to assess the exudative change-free percentage of the eyes treated with aflibercept or ranibizumab. Recurrence presenting with retinal fluid as determined using OCT was considered as failure. Differences between aflibercept and ranibizumab were evaluated with the log rank test. Bivariate relationships were examined using the Spearman's correlation coefficient. A $P$-value of $<0.05$ was considered statistically significant.

\section{Results \\ Participants}

Table 1 summarizes the baseline clinical characteristics of the treatment-naïve AMD eyes treated with ranibizumab $(\mathrm{N}=77)$ and aflibercept $(\mathrm{N}=71)$, and converted from ranibizumab to aflibercept ( $\mathrm{N}=88)$. Age, sex, VA, GLD, and CRT showed no statistically significant differences between these groups of treatment-naïve AMD eyes. With aflibercept treatment, the preinjection VAs of the treatment-naïve eyes were better than those of the converted group $(P<0.001)$. The converted group included a higher percentage with PCV $(P=0.039)$. The mean number of subsequent injections for 12 months was 4.9 with aflibercept (4.4 with treatment-naïve eyes and 5.7 with eyes converted from ranibizumab) and 4.4 with ranibizumab.

Table I Baseline clinical characteristics of AMD patients who received intravitreal injection of aflibercept or ranibizumab

\begin{tabular}{llll}
\hline Characteristics & Treatment-naïve & & \multicolumn{1}{c}{$\begin{array}{c}\text { Conversion from } \\
\text { ranibizumab to aflibercept }\end{array}$} \\
\cline { 2 - 4 } & Ranibizumab & Aflibercept & $88(80)$ \\
\hline Eyes (cases) & $77(72)$ & $71(66)$ & $77.9 \pm 7.7$ \\
Age, mean \pm SD (years) & $77.3 \pm 9.1$ & $77.0 \pm 8.4$ & $59(67)$ \\
Sex, $\mathrm{n}(\%)$ & & & $29(33)$ \\
$\quad$ Male & $47(67)$ & $51(72)$ & $0.54 \pm 0.44 *$ \\
$\quad$ Female & $30(39)$ & $20(28)$ & $4,448.4 \pm 1,717.3$ \\
LogMAR VA, mean \pm SD & $0.62 \pm 0.42$ & $0.52 \pm 0.51$ & $403.6 \pm 151.2$ \\
GLD, mean \pm SD $(\mu \mathrm{m})$ & $3,181.9 \pm 1,500.8$ & $3,687.6 \pm 1,539.6$ & $26(30)$ \\
CRT, mean \pm SD $(\mu \mathrm{m})$ & $414.6 \pm 171.6$ & $401.6 \pm 178.4$ & $57(65)^{*}$ \\
Subtype, $\mathrm{n}(\%)$ & & & $5(6)$ \\
$\quad$ Typical AMD & $35(46)$ & $35(49)$ & $33(47)$ \\
PCV & $38(49)$ & $3(4)$ & \\
RAP & $4(5)$ & & \\
\hline
\end{tabular}

Note: $* P<0.05$.

Abbreviations: AMD, age-related macular degeneration; logMAR VA, logarithm of the minimal angle of resolution visual acuity; GLD, greatest linear dimension; CRT, central retinal thickness; PCV, polypoidal choroidal vasculopathy; RAP, retinal angiomatous proliferation; SD, standard deviation. 


\section{Response/dependence and initial} recurrence in treatment-naïve AMD eyes

Response/dependence to the induction therapies with aflibercept and ranibizumab of the treatment-naïve AMD eyes is shown in Figure 1A and B, respectively. Among the 71 eyes treated with aflibercept, $54(76 \%)$ had a good response, but 20 of these $(37 \%)$ were aflibercept-dependent. We recommended proactive bimonthly aflibercept treatments for poorly responding eyes and well-responding but dependent eyes. The patients selected the recommended treatment except for 2 patients who chose to continue on successive bimonthly treatments; therefore the two eyes of those patients could not be judged for dependence.

Figure 1C shows the exudative change-free survival percentages of treatment-naïve AMD eyes after aflibercept or ranibizumab treatment which were good responders according to Kaplan-Meier survival analyses. The eyes treated with ranibizumab had a relapse within 1 month after the completion of induction therapy, while the eyes treated with aflibercept started to relapse between 1 and 2 months after the completion of induction therapy. The cumulative incidences of recurrences in the eyes treated with aflibercept and ranibizumab were $38 \%$ and $51 \%$ at 3 months post-treatment, respectively, and $81 \%$ and $87 \%$ at 12 months posttreatment, respectively. There was no statistical difference in the survival percentage between the two groups $(P=0.263)$.

\section{Effect of aflibercept on eyes in the converted group}

We also investigated the effect of aflibercept induction therapy on the eyes converted from ranibizumab treatment. Of the 38 eyes that responded well but were dependent
A

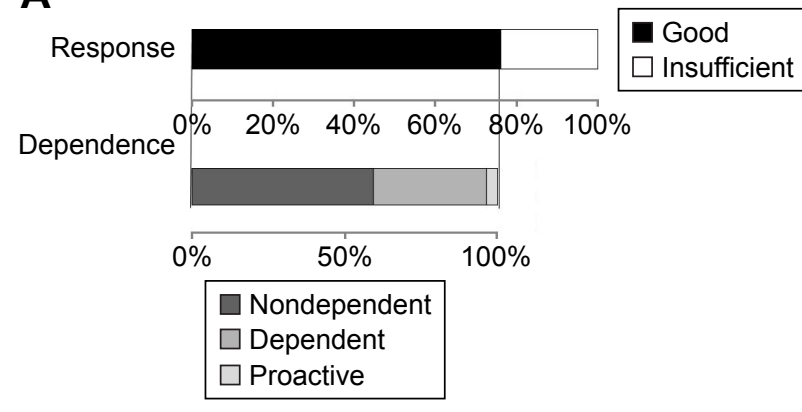

B

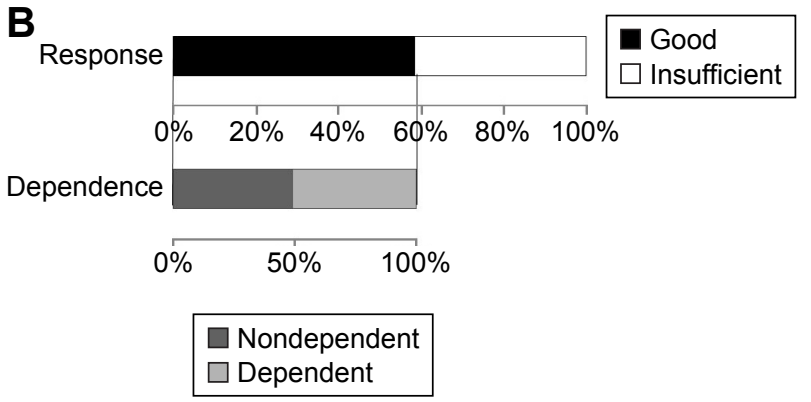

D

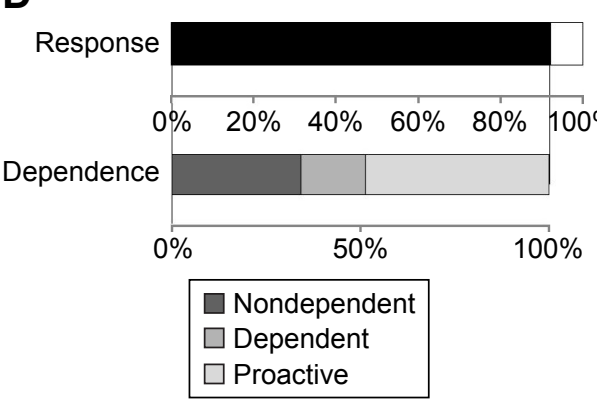

C

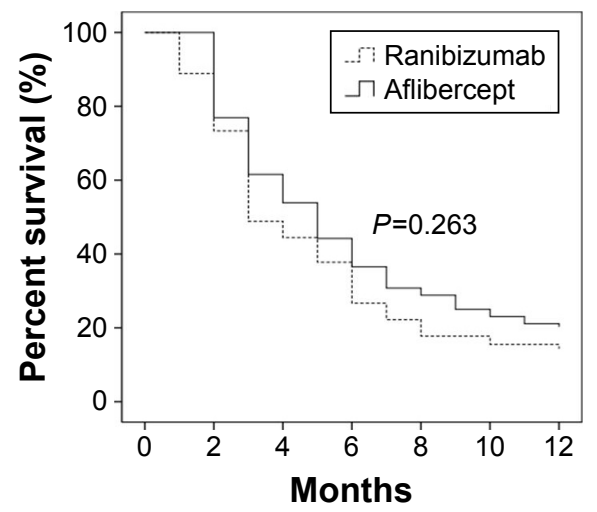

E

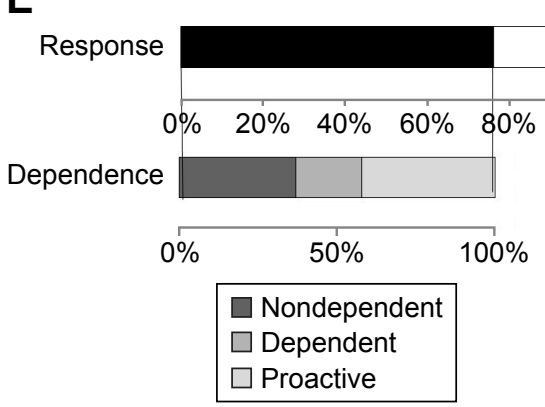

Figure I Response and dependence of eyes with age-related macular degeneration treated with aflibercept (A) and ranibizumab (B) are shown. Exudative change-free survival percentage of age-related macular degeneration patients treated with aflibercept or ranibizumab according to the Kaplan-Meier survival model is presented. In (C), the horizontal axis shows time from the completion of the induction therapy, and the vertical axis shows the percentages of eyes that showed no recurrence. Response to and dependence on aflibercept of eyes with age-related macular degeneration that were converted from ranibizumab treatment are shown: eyes with a good response having required repeated injections of (dependent on) ranibizumab (D) and eyes with an insufficient response to ranibizumab (E). 
Table 2 Correlation coefficient between each factor and response/dependence in AMD patients

\begin{tabular}{lllllllllll}
\hline & Age & Sex & Subtype & VA & CRT & GLD & PED & IRF & SRF & Treatment history \\
\hline Response & & & & & & & & & & \\
$\quad r$ & 0.040 & 0.148 & -0.141 & -0.035 & -0.035 & 0.096 & 0.017 & -0.010 & 0.096 & 0.117 \\
$\quad P$ & 0.613 & 0.063 & 0.076 & 0.662 & 0.664 & 0.232 & 0.834 & 0.901 & 0.228 & 0.144 \\
$\begin{array}{l}\text { Dependence } \\
\quad\end{array}$ & -0.018 & -0.061 & -0.008 & 0.027 & -0.043 & -0.035 & 0.222 & -0.221 & 0.188 & 0.058 \\
$\quad P$ & 0.863 & 0.559 & 0.938 & 0.795 & 0.677 & 0.738 & $0.031^{*}$ & $0.031^{*}$ & 0.067 & 0.577 \\
\hline
\end{tabular}

Notes: Treatment history: naïve or converted from ranibizumab. $* P<0.05$.

Abbreviations: AMD, age-related macular degeneration; VA, visual acuity; CRT, central retinal thickness; GLD, greatest linear dimension; PED, pigment epithelial detachment; IRF, intraretinal fluid; SRF, subretinal fluid.

on ranibizumab, 35 (92\%) had a good response to aflibercept induction therapy. Although all the eyes in the converted group were recommended for proactive treatment, patients with a total of 18 eyes $(51 \%)$ selected the PRN regimen, and six of the 18 eyes (33\%) were afliberceptdependent (Figure 1D). Figure 1E shows that of the 50 eyes that responded insufficiently to ranibizumab, 38 (76\%) responded well to aflibercept. Again, although the patients were recommended for proactive treatment, patients with a total of 22 of the 38 eyes (58\%) selected the PRN regimen, and eight of these eyes (36\%) were aflibercept-dependent. Among the patients in the converted group, patients with a total of 56 of the 88 eyes (64\%) followed the recommended treatment.

\section{Preinjection factors}

For the aflibercept-treated group, we studied the correlation coefficients between each preinjection factor and response or dependence to treatment (Table 2). There was a correlation between dependence and PED $(r=0.222 ; P=0.031)$, and IRF $(r=-0.221 ; P=0.031)$. Other factors (age, sex, disease subtype, VA, CRT, GLD, SRF, and treatment history) had no correlation with response or dependence.

\section{Visual outcomes and CRT}

Figure 2A shows that treatment-naïve aflibercept-treated eyes achieved significantly better VA than the baseline VA over 12 months of the treatment $(P<0.001)$ and that the converted group gained significantly improved VA at 6 and 9 months during the treatment, but could not retain the improved VA at 12 months. More detailed analyses showed that the VA of the PRN treatment group (good response/non-dependent) improved at 3 and 6 months during the treatment, and then worsened at 9 and 12 months. In contrast, the VA of the proactive treatment group improved significantly from 6 to 12 months, and the mean VA was continuously maintained (Figure 2B).

The CRT values of the treatment-naïve eyes treated with aflibercept and the eyes converted from ranibizumab to aflibercept were 401.6 and $403.6 \mu \mathrm{m}$, respectively, at baseline. The mean CRT values then decreased to 259.0 and $269.4 \mu \mathrm{m}$, respectively, at 3 months and remained stable
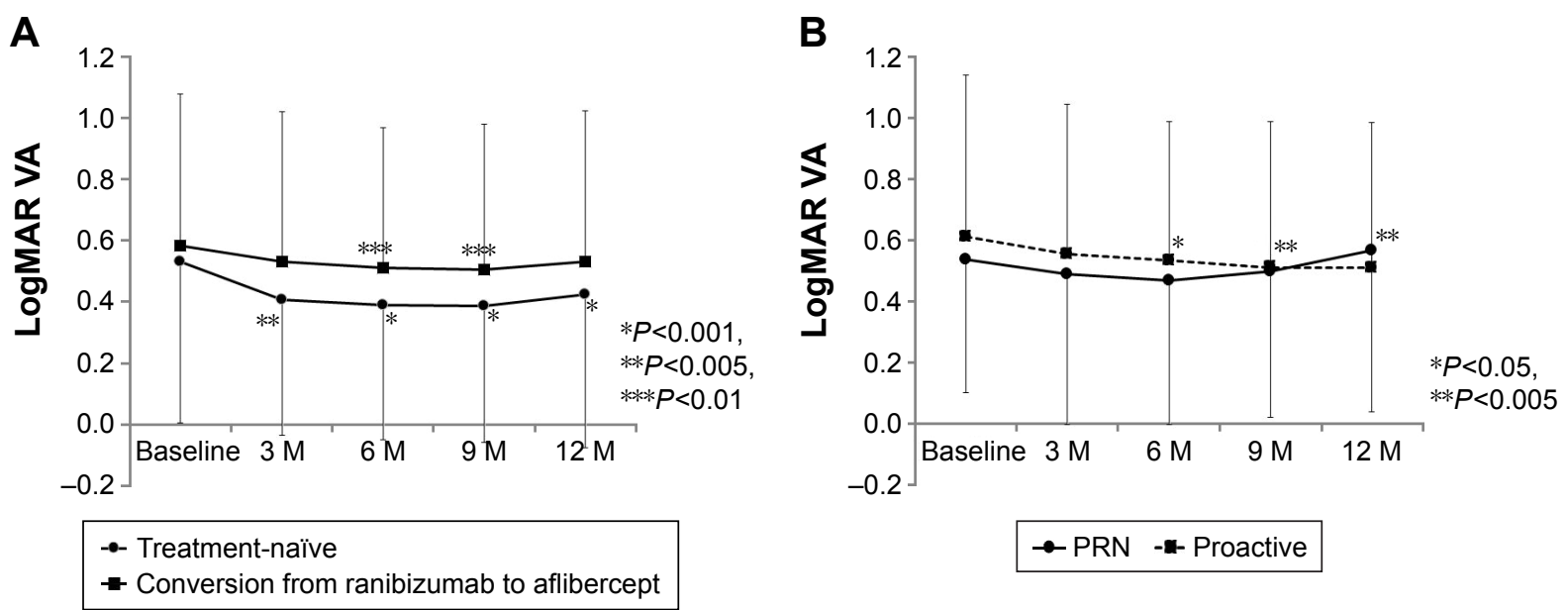

Figure 2 Mean best-collected VA \pm standard deviation from baseline to 12 months after intravitreal injections of aflibercept for naïve age-related macular degeneration eyes and for eyes converted from ranibizumab to aflibercept $(\mathbf{A})$, and the VA of the PRN- and proactive-treated eyes converted from ranibizumab to aflibercept (B).

Note: Asterisks indicate significant differences compared with baseline and between both the groups.

Abbreviations: VA, visual acuity; PRN, pro re nata; logMAR, logarithm of the minimal angle of resolution; $M$, months. 
A

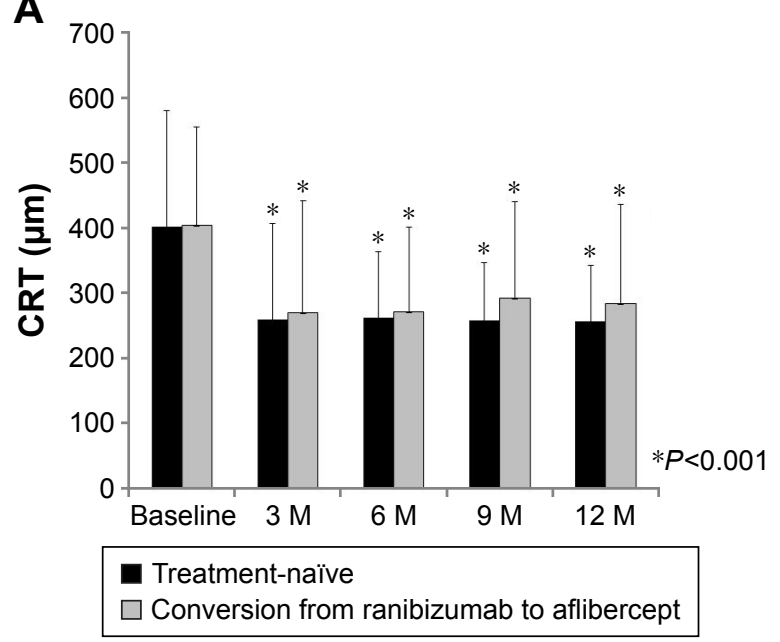

B

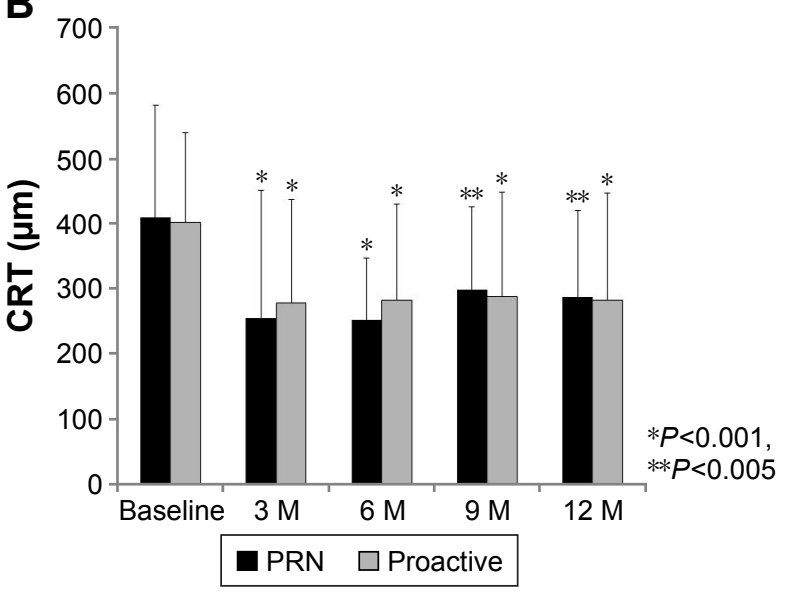

Figure 3 Mean CRT \pm standard deviation from baseline to 12 months after intravitreal injections of aflibercept for naïve age-related macular degeneration eyes and for eyes converted from ranibizumab to aflibercept (A), and the CRT of the PRN- and proactive-treated eyes converted from ranibizumab to aflibercept (B).

Note: Asterisks indicate a significant difference compared with baseline and between both the groups.

Abbreviations: CRT, central retinal thickness; PRN, pro re nata; $M$, months.

until 12 months of the treatment period (Figure 3A). Both the treatments reduced CRT significantly over 12 months of treatment $(P<0.001)$. The CRT of the eyes that received proactive treatment and PRN treatment are also shown separately in Figure 3B. The CRT of both the groups decreased significantly from 3 to 12 months.

\section{Discussion}

Previous studies using anti-VEGF therapy for AMD patients have indicated that anti-VEGF therapy is symptomatic, and the strict maintenance of a dry macula during the maintenance phase is essential to retain improved vision. Furthermore, maintenance of a dry macula can be achieved only by frequent monitoring and/or treatment. Thus, one major issue in clinical practice involves knowing the patients' responses to and dependence on anti-VEGF drugs to determine who will need to continue treatment, and at what interval by also considering the feasibility of the treatment in clinical practice. For this purpose, a treat-and-extend approach would be one good choice, ${ }^{16}$ but sometimes it could result in an overtreatment irrespective of choroidal neovascularization (CNV) activity. The data showed that approximately $63 \%, 38 \%$, and $19 \%$ of the treatment-naïve AMD eyes did not require additional aflibercept injections during the following 3, 6, and 12 months of treatment, respectively (Figure 1C). Using Kaplan-Meier survival analyses, the eyes treated with ranibizumab were shown to relapse earlier than the aflibercept-treated eyes, but the overall recurrence percentage was not statistically different between these drug groups as reported recently by Inoue et al, ${ }^{17}$ and more than $80 \%$ of the treated eyes experienced a recurrence within 1 year.
Thus, with the use of aflibercept, more than $80 \%$ of the patients needed additional injections within 1 year, and approximately half of the patients needed proactive treatment due to either a poor response or dependence.

It has been reported that aflibercept therapy is beneficial for AMD patients who exhibit recurrent or resistant exudative changes following multiple injections with either bevacizumab or ranibizumab. ${ }^{7-12} \mathrm{We}$ also found a good response to aflibercept in the group converted from ranibizumab treatment. Although proactive treatment had been recommended for the converted patients, about half of the patients selected the PRN treatment; these patients had already undergone repeated injections, and therefore, their expectations for treatment efficacy may not have been high. Among those who selected the PRN regimen, aflibercept dependence recurred in $35 \%$ of those with good-responding eyes, but this dependence percentage was the same as that of the treatment-naïve AMD eyes. These results suggest that it may not be necessary to distinguish eyes based on their history of previous anti-VEGF therapy (Figure 1A, D, and E), and it may be possible to start the maintenance phase using the PRN regimen to test for dependence in all the converted cases.

In the ranibizumab-treated group, we observed a significant VA improvement at 3 months (logMAR VA, 0.49; $P<0.005)$ and 6 months $(\log$ MAR VA, $0.50 ; P<0.005)$ compared to the baseline (logMAR VA, 0.62 ), but these eyes could not maintain improved VA at 9 months (logMAR VA, $0.56)$ and 12 months (logMAR VA, 0.55) with PRN treatment, probably because of undertreatment and insufficient dry macula in the clinical setting. The mean number of 
subsequent ranibizumab injections for 12 months was 4.4 , which was also a smaller number compared with that of previous reports. ${ }^{1,3,14}$ To avoid the risk of undertreatment in these PRN patients, we adopted the proactive bimonthly injections following the 3-month induction therapy using aflibercept for treatment-naïve eyes that had responded insufficiently or had experienced early relapse (dependent). This resulted in more frequent injections of aflibercept (average of 4.9 times/year) than those of the ranibizumab-treated group, but were still less than those performed in the PrONTO study (average of 5.6 times/year). ${ }^{14}$ Using this protocol, naïve AMD eyes $(\mathrm{N}=71)$ maintained a significantly better VA from baseline up to 1 year, implying that intensive treatment of insufficient responders $(\mathrm{N}=17)$ and good responders/dependent $(\mathrm{N}=18)$, either of them reaching up to $49 \%$ of the total, on average, was effective. In the SEVEN-UP study on ranibizumab-treated patients, ${ }^{18}$ Rofagha et al reported that 30 of the 65 study eyes $(46 \%)$ were receiving ongoing ocular anti-VEGF treatments. Likewise, approximately half of the aflibercept-treated eyes were considered to need intensive treatments, similar to approximately half of the ranibizumab-treated eyes.

The converted group included a higher percentage with PCV (65\%), which indicated that eyes with PCV may have a lower response or a higher dependence on ranibizumab. In this group, the VA of the proactive treatment group improved significantly from 6 to 12 months during the treatment (Figure 2B), suggesting that even if a patient responded poorly or required multiple injections of ranibizumab, proactive bimonthly treatment with aflibercept could still improve the VA. However, $36 \%$ of the patients who selected the PRN regimen could not retain the improved VA in the maintenance phase. Furthermore, some of the patients on the PRN regimen in the converted group did not desire treatment when recommended, and were therefore very likely undertreated. A limitation of this study is the retrospective study design with no case controls, and the number of patients was small. In addition, the eyes without follow-up of 12 months were excluded, and these patients may not have returned because of poorer VA, potentially biasing the outcomes; however, the response and dependence could be useful parameters to consider further plans for anti-VEGF therapies.

Aflibercept binds to members of the VEGF family, including all VEGF-A and VEGF-B isoforms, as well as to $\mathrm{PlGF},{ }^{4}$ whereas ranibizumab blocks the receptor-binding domain of all isoforms of VEGF-A. ${ }^{19}$ Previous reports confirmed the presence of PlGF by immunohistochemistry of human choroidal neovascular membranes,${ }^{20}$ and PlGF mRNA expression was found to be significantly upregulated during the course of experimental CNV.${ }^{21}$ It is well known that PlGF binds to the VEGF receptor-1 (VEGFR-1) and is involved not only in ocular angiogenesis but also in ocular permeability. A previous report also demonstrated that PlGF-1 regulated the external blood-retinal barrier (BRB) by a direct effect on retinal pigment epithelial tight cell junctions through VEGFR-1.22 It is also known that the BRB, which consists of inner and outer components, is a physiological barrier that regulates ions, proteins, and water flux into and out of the retina. Neovascular AMD is thought to be a result of an alteration of the outer BRB. ${ }^{23}$ Simultaneous inhibition of multiple components of the VEGF family may improve AMD, and the differences between aflibercept and ranibizumab in biological activity and binding affinities may account for resolution of the fluid. Recent studies reported that the subfoveal choroidal thickness decreased significantly in eyes with AMD during 3 months of aflibercept treatment, ${ }^{24,25}$ but remained unchanged in ranibizumab-treated eyes. ${ }^{24}$ It was also reported that aflibercept was effective in reducing PED that was refractory or exhibited tachyphylaxis to other antiVEGF drugs, ${ }^{26,27}$ although not all the patients had altered PED after switching to aflibercept. ${ }^{26}$ The higher percentages of dry macula, decreased subfoveal choroidal thickness, and reduced PED in the aflibercept-treated group may be related to the pharmacological characteristics of aflibercept. Our results showed that there was a correlation between dependence and PED $(r=0.222 ; P=0.031)$ and IRF ( $r=-0.221$; $P=0.031)$. Although aflibercept was effective in reducing $\mathrm{PED}$, frequent injections may be necessary for eyes with PED. Eyes with IRF may also need frequent injections.

In conclusion, induction therapy using aflibercept resulted in a good response of $76 \%$ in treatment-naïve patients, but $37 \%$ of these well-responding eyes relapsed within 3 months, and more than $80 \%$ of the eyes relapsed within 1 year. Approximately half of the treatment-naïve patients were recommended proactive bimonthly treatment. By recommending proactive bimonthly treatment for the eyes that were poorly responding or well responding but dependent, we could still substantially improve VA, indicating that these are clear and reasonable parameters for directing anti-VEGF therapy protocols.

\section{Disclosure}

The authors report no conflicts of interest in this work.

\section{References}

1. Brown DM, Kaiser PK, Michels M, et al; ANCHOR Study Group. Ranibizumab versus verteporfin for neovascular age-related macular degeneration. N Engl J Med. 2006;355(14):1432-1444. 
2. CATT Research Group; Martin DF, Maguire MG, Ying GS, Grunwald JE, Fine SL, Jaffe GJ. Ranibizumab and bevacizumab for neovascular age-related macular degeneration. $N$ Engl J Med. 2011; 364(20):1897-1908.

3. Rosenfeld PJ, Brown DM, Heier JS, et al; MARINA Study Group. Ranibizumab for neovascular age-related macular degeneration. $N$ Engl J Med. 2006;355(14):1419-1431.

4. Holash J, Davis S, Papadopoulos N, et al. VEGF-Trap: a VEGF blocker with potent antitumor effects. Proc Natl Acad Sci U S A. 2002;99(17): 11393-11398.

5. Papadopoulos N, Martin J, Ruan Q, et al. Binding and neutralization of vascular endothelial growth factor (VEGF) and related ligands by VEGF Trap, ranibizumab and bevacizumab. Angiogenesis. 2012;15(2): 171-185.

6. Stewart MW, Rosenfeld PJ. Predicted biological activity of intravitreal VEGF Trap. Br J Ophthalmol. 2008;92(5):667-668.

7. Bakall B, Folk JC, Boldt HC, et al. Aflibercept therapy for exudative age-related macular degeneration resistant to bevacizumab and ranibizumab. Am J Ophthalmol. 2013;156(1):15-22.e1.

8. Cho H, Shah CP, Weber M, Heier JS. Aflibercept for exudative AMD with persistent fluid on ranibizumab and/or bevacizumab. $\mathrm{Br} J$ Ophthalmol. 2013;97(8):1032-1035.

9. Ferrone PJ, Anwar F, Naysan J, et al. Early initial clinical experience with intravitreal aflibercept for wet age-related macular degeneration. Br J Ophthalmol. 2014;98 Suppl 1:i17-i21.

10. Kumar N, Marsiglia M, Mrejen S, et al. Visual and anatomical outcomes of intravitreal aflibercept in eyes with persistent subfoveal fluid despite previous treatments with ranibizumab in patients with neovascular agerelated macular degeneration. Retina. 2013;33(8):1605-1612.

11. Messenger WB, Campbell JP, Faridi A, et al. Injection frequency and anatomic outcomes 1 year following conversion to aflibercept in patients with neovascular age-related macular degeneration. Br J Ophthalmol. 2014;98(9):1205-1207.

12. Wykoff CC, Brown DM, Maldonado ME, Croft DE. Aflibercept treatment for patients with exudative age-related macular degeneration who were incomplete responders to multiple ranibizumab injections (TURF trial). Br J Ophthalmol. 2014;98(7):951-955.

13. Heier JS, Brown DM, Chong V, et al; VIEW 1 and VIEW 2 Study Groups. Intravitreal aflibercept (VEGF trap-eye) in wet age-related macular degeneration. Ophthalmology. 2012;119(12):2537-2548.

14. Lalwani GA, Rosenfeld PJ, Fung AE, et al. A variable-dosing regimen with intravitreal ranibizumab for neovascular age-related macular degeneration: year 2 of the PrONTO Study. Am J Ophthalmol. 2009; 148(1):43-58.e1.

15. Yanuzzi LA. Retinal histology. In: The Retinal Atlas. Philadelphia, PA: Saunders; 2010:2-4
16. Arnold JJ, Campain A, Barthelmes D, et al; Fight Retinal Blindness Study Group. Two-year outcomes of "treat and extend" intravitreal therapy for neovascular age-related macular degeneration. Ophthalmology. 2015;122(6):1212-1219.

17. Inoue M, Yamane S, Sato S, Sakamaki K, Arakawa A, Kadonosono K. Comparison of time to retreatment and visual function between ranibizumab and aflibercept in age-related macular degeneration. Am J Ophthalmol. 2016;169:95-103.

18. Rofagha S, Bhisitkul RB, Boyer DS, Sadda SR, Zhang K; SEVEN-UP Study Group. Seven-year outcomes in ranibizumab-treated patients in ANCHOR, MARINA, and HORIZON: a multicenter cohort study (SEVEN-UP). Ophthalmology. 2013;120(11):2292-2299.

19. Ferrara N, Damico L, Shams N, Lowman H, Kim R. Development of ranibizumab, an anti-vascular endothelial growth factor antigen binding fragment, as therapy for neovascular age-related macular degeneration. Retina. 2006;26(8):859-870.

20. Otani A, Takagi H, Oh H, et al. Vascular endothelial growth factor family and receptor expression in human choroidal neovascular membranes. Microvasc Res. 2002;64(1):162-169.

21. Rakic JM, Lambert V, Devy L, et al. Placental growth factor, a member of the VEGF family, contributes to the development of choroidal neovascularization. Invest Ophthalmol Vis Sci. 2003;44(7):3186-3193.

22. Miyamoto N, de Kozak Y, Jeanny JC, et al. Placental growth factor-1 and epithelial haemato-retinal barrier breakdown: potential implication in the pathogenesis of diabetic retinopathy. Diabetologia. 2007; 50(2):461-470.

23. Cunha-Vaz J, Bernardes R, Lobo C. Blood-retinal barrier. Eur J Ophthalmol. 2010;21 Suppl 6:S3-S9.

24. Gharbiya M, Cruciani F, Mariotti C, Grandinetti F, Marenco M, Cacace V. Choroidal thickness changes after intravitreal antivascular endothelial growth factor therapy for age-related macular degeneration: ranibizumab versus aflibercept. J Ocul Pharmacol Ther. 2015;31(6): 357-362.

25. Koizumi H, Kano M, Yamamoto A, et al. Short-term changes in choroidal thickness after aflibercept therapy for neovascular age-related macular degeneration. Am J Ophthalmol. 2015;159(4):627-633.

26. Patel KH, Chow CC, Rathod R, et al. Rapid response of retinal pigment epithelial detachments to intravitreal aflibercept in neovascular age-related macular degeneration refractory to bevacizumab and ranibizumab. Eye (Lond). 2013;27(5):663-667; quiz 668.

27. Broadhead GK, Hong T, Zhu M, et al. Response of pigment epithelial detachments to intravitreal aflibercept among patients with treatmentresistant neovascular age-related macular degeneration. Retina. 2015; 35(5):975-981.
Clinical Ophthalmology

\section{Publish your work in this journal}

Clinical Ophthalmology is an international, peer-reviewed journal covering all subspecialties within ophthalmology. Key topics include: Optometry; Visual science; Pharmacology and drug therapy in eye diseases; Basic Sciences; Primary and Secondary eye care; Patient Safety and Quality of Care Improvements. This journal is indexed on Submit your manuscript here: http://www.dovepress.com/clinical-ophthalmology-journal
Dovepress

PubMed Central and CAS, and is the official journal of The Society of Clinical Ophthalmology (SCO). The manuscript management system is completely online and includes a very quick and fair peer-review system, which is all easy to use. Visit http://www.dovepress.com/ testimonials.php to read real quotes from published authors. 